\title{
Planejamento Interdisciplinar Para Reabilitação Oral: Um Relato de Caso de Protocolo Bränemark
}

\section{Interdisciplinary Planning for the Oral Rehabilitation: Branemark'S Clinic Case Report}

\author{
Clóvis Lamartine de Moraes Melo Netoa*; Rodrigo Lorenzi Poluhab; Silvia Sbeghen Sábio ${ }^{\mathrm{b}}$; Juliana Squizatto Leite; \\ Sérgio Sábio
}

\author{
aUniversidade Estadual de Maringá, Pós-Graduação Latu Sensu em Prótese Dentária, PR, Brasil \\ bUniversidade Estadual de Maringá, Curso de Odontologia, PR, Brasil \\ *E-mail: clovislamartine@hotmail.com \\ Recebido: 17 de março de 2015; Aceito: 21 de julho de 2015
}

\begin{abstract}
Resumo
A reabilitação oral em pacientes edêntulos é um desafio que a odontologia atual vem tentando superar com implantes osseointregrados. O fator socioeconômico ainda é uma das causas de maior dificuldade para o tratamento em pacientes edêntulos. No presente relato de caso, será descrita a associação entre projeto de extensão e atendimentos interdisciplinares odontológicos para a reabilitação de um paciente com baixa condição financeira. Este trabalho relata o caso de um paciente de 55 anos que se queixava de sensibilidade em alguns dentes, desconforto com a prótese removível inferior, dificuldades mastigatórias, fonéticas, assim como, estética insatisfatória. Com o objetivo de restabelecer função e estética para uma melhor qualidade de vida do paciente, um tratamento envolvendo várias áreas da odontologia foi realizado. Após avaliação de uma equipe interdisciplinar e posterior discussão com o paciente sobre os possíveis planos de tratamento, realizou-se a exodontia dos dentes inferiores e instalação de cinco implantes. Para reabilitação protética, realizou-se a confecção do protocolo Bränemark na arcada inferior, que apresentava pouco rebordo ósseo e de uma nova prótese total superior. Ao final foi evidente a melhora estética e funcional obtida com o tratamento, tendo o paciente recuperado a autoestima e a vontade de cuidar de sua saúde bucal. A partir desse relato de caso pode-se observar que o protoloco Bränemark é uma indicação viável no tratamento de pacientes edêntulos e com pouco rebordo alveolar por conferir estabilidade à prótese.
\end{abstract}

Palavras-chave: Implantação Dentária. Prótese Total. Prótese Dentária.

\begin{abstract}
Oral rehabilitation in edentulous patients is a challenge that the current dentistry has been trying to overcome through osseointegrated implants. The socio-economic factor is still a cause of the greatest difficulty for treatment in edentulous patients. In this case report we describe the association between extension project and interdisciplinary care for the rehabilitation of a patient with low financial condition. This study reports the case of a 55 year-old patient who complained about sensitivity in some teeth, discomfort with mandibular removable partial denture, masticatory and phonetic difficulties, as well as poor aesthetics. The purpose of restoring function and aesthetics for a better life quality of the patient, a treatment involving various dental specialties was conducted. After the evaluation of a interdisciplinary team and further discussion with the patient about possible treatment plans, it was decided to extract the mandibular teeth and placement of five dental implants. For the prosthetic rehabilitation Bränemark protocol was made on the mandibular region which had little bony ridge, and a new maxillary removable complete denture. In the end of the treatment there was evident aesthetic and functional improvement and the patient regained self-esteem and the will to care of his oral health. Based on this case report it can be noted that Branemark protocol is a viable choice for the treatment of edentulous patients and it is indicated in cases that there is little alveolar ridge to confer prosthesis stability.
\end{abstract}

Keywords: Dental Implantation. Denture, Complete. Dental Prosthesis.

\section{Introdução}

O levantamento epidemiológico SB Brasil 2010 demonstrou que $23,9 \%$ dos idosos (65-74 anos) necessitam de prótese total em pelo menos uma arcada dentária e 15,4\% necessitam de prótese total tanto na arcada superior quanto na arcada inferior. Esse levantamento possibilitou visualizar que há uma redução da necessidade de próteses em adolescentes e adultos $^{1}$. Entretanto, os idosos ainda continuam necessitando de atenção, visto que usuários de próteses totais apresentam uma grande limitação representada pela perda óssea decorrente do contínuo processo de reabsorção, comprometendo a retenção e estabilidade destas. Nesse sentido, a substituição de tecidos bucais perdidos por meio de próteses totais consiste em um problema tanto para o cirurgião-dentista quanto para o paciente.

Os implantes osseointegrados vêm sendo utilizados para tratamento de pacientes com ausência total ou parcial de dentes. Quando se tem volume e densidade óssea adequados pode se obter previsibilidade e sucesso clínico ${ }^{2}$. Para garantir a segurança cirúrgica em casos de reabilitações com implantes em mandíbula, é imprescindível a identificação espacial de acidentes anatômicos, como o forame mentoniano e o canal mandibular. Além disso, a espessura óssea ideal deve ser de no mínimo cinco milímetros. Dessa forma, o diagnóstico por imagens é tão importante quanto um efetivo 
exame clínico para o planejamento cirúrgico ${ }^{3}$. Uma opção para tratamento de pacientes desdentados com espessura alveolar inferior à necessária é o Protocolo Bränemark, que consiste na reabilitação de desdentados inferiores, nos quais são colocados de quatro a seis implantes entre os forames mentonianos, por não possuírem disponibilidade óssea na região posterior de mandíbula. Os implantes são mantidos em repouso por um período de quatro meses, dependendo do tipo de implante colocado e a qualidade do tecido ósseo. Após isso, passam a dar sustentação a uma prótese fixa, confeccionada a partir de uma estrutura metálica que se estende além do forame, sobre a qual são prensados dentes de resina acrílica ${ }^{4}$. Apesar dessa explicação simplista, o correto planejamento assume papel fundamental no sucesso do tratamento. Diversos fatores devem ser levados em consideração. Fatores como a passividade são de extrema importância em próteses protocolo Bränemark. Forças mastigatórias normais podem comprometer a osseointegração, quando a estrutura metálica não apresenta boa adaptação e passividade ${ }^{5,6}$. O posicionamento do implante também pode determinar o sucesso do tratamento. Uma boa estética e funcionalidade depende do correto posicionamento do implante. Esse fator só é estabelecido quando se planeja um guia de orientação bem posicionado.

O objetivo deste relato de caso é mostrar a integração multiprofissional de várias especialidades odontológicas, por meio de planejamentos, tratamentos e com associação de um projeto de extensão da Universidade Estadual de Maringá, para a reabilitação oral de um paciente de baixa condição financeira.

\section{Relato de Caso Clínico}

Paciente do sexo masculino, 55 anos de idade, procurou atendimento odontológico na Universidade Estadual de Maringá para um tratamento reabilitador, queixando-se de sensibilidade na região dos incisivos, caninos e pré-molares, referentes aos dentes inferiores, desconforto com a prótese parcial fixa (PPR) inferior, dificuldades mastigatórias, fonéticas e insatisfação com a estética de seu sorriso. Ele também fazia uso de uma prótese total superior. Durante a anamnese, o paciente relatou que era fumante e as respostas referentes às informações sobre a sua saúde sistêmica retratavam normalidade.

Inicialmente, o paciente passou por avaliação com um periodontista, que fez uma adequação do meio bucal e posteriormente com um protesista, cirurgião buco-maxilofacial, ortodontista e endodontista, então houve a elaboração de possibilidades de tratamento. No exame intrabucal foram observadas áreas relativas a abfrações cervicais vestibulares, doença periodontal, destacando as grandes perdas ósseas em sua maioria horizontais, presença de bolsa periodontal em todos os dentes, recessões gengivais com mais de três milímetros, mobilidades dentárias acentuadas nos incisivos inferiores de grau 3 e caninos e pré-molares com grau 1 . Baseando-se na avaliação periodontal, os incisivos centrais e laterais foram considerados dentes perdidos, enquanto os caninos e pré-molares, duvidosos.

O paciente utilizava prótese total $(\mathrm{PT})$ superior há 10 anos e não fazia uso de PPR inferior devido à dificuldade de se acostumar a esta. Devido à ausência de dentes posteriores inferiores, a alimentação ficava concentrada quase que exclusivamente na região anterior da cavidade bucal. Dessa forma, era possível visualizar uma reabsorção no rebordo superior anterior e hiperplasia inflamatória, oriunda dessa mastigação anteriorizada (síndrome da combinação). A PT superior apresentava sorriso invertido, diastema entre os incisivos centrais (preferência do paciente), zênites dos dentes em acrílico não seguiam uma boa harmonia, obviamente as giroversões e má posições dos dentes inferiores contribuíam para esse quadro clínico (Figura 1).

Com o diagnóstico de periodontite crônica e diante da recusa do paciente em realizar a reabilitação com prótese parcial removível inferior, o tratamento proposto consistia na exodontia dos incisivos centrais, laterais e na tentativa de preservação de caninos e pré-molares, com a realização de raspagem e alisamento radicular nestes elementos. A avaliação endodôntica constatou tratamento endodôntico satisfatório no dente 35 para reabilitação protética.

Posteriormente com o controle da saúde gengival e óssea, as posições das coroas e estética dos dentes remanescentes poderiam ser corrigidas com ortodontia e dentística (clareamento, restaurações de resina composta para cobrir raízes expostas e reanatomizações). Outra opção seria reabilitar os dentes restantes com tratamento endodôntico seguido de coroas em porcelana (com núcleos metálicos fundidos, caso necessário). Independentes da escolha do tratamento seguidamente seriam instalados implantes nos espaços protéticos e próteses implanto-suportadas nessas áreas. Para o arco superior foi descartada a reabilitação com implantes por questões financeiras. Em virtude da baixa condição financeira do paciente e da incerteza do sucesso pelo longo prazo do tratamento periodontal e o longo período de tratamento, foi sugerida outra possibilidade reabilitadora, referente ao protocolo de Branemark, mediante extração de todos os dentes inferiores.

Uma overdenture implanto-suportada (para o arco inferior) foi proposta como alternativa reabilitadora por causa da facilidade durante a higienização, mas o paciente optou por uma prótese sobre implantes fixa (Protocolo de Bränemark). A formação do biofilme parece ser crítica para o desenvolvimento das doenças peri-implantares e pode ser responsável pela alteração na biocompatibilidade da superfície do implante, podendo levar a sua perda 7,8,9. A relação de causa-efeito entre a placa dental e o desenvolvimento de mucosite é similar ao modelo de 
desenvolvimento de gengivite ${ }^{10}$. De modo que se acredita que a mucosite seja a precursora da peri-implantite, da mesma forma que a gengivite é a precursora da periodontite ${ }^{8}$. Há, também, fortes evidências de que a má higiene bucal, o histórico de doença periodontal e o uso de tabaco são fatores de risco para o desenvolvimento dessas doenças ${ }^{11-12}$. $\mathrm{O}$ paciente foi orientado quanto à necessidade de realizar a higiene bucal de forma cuidadosa e rigorosa, além de tentar reduzir o consumo de tabaco, prevenindo, assim, essas doenças e a perda dos implantes.

Figura 1: Foto inicial e RX Panorâmico do paciente mostrando quadro clínico complexo
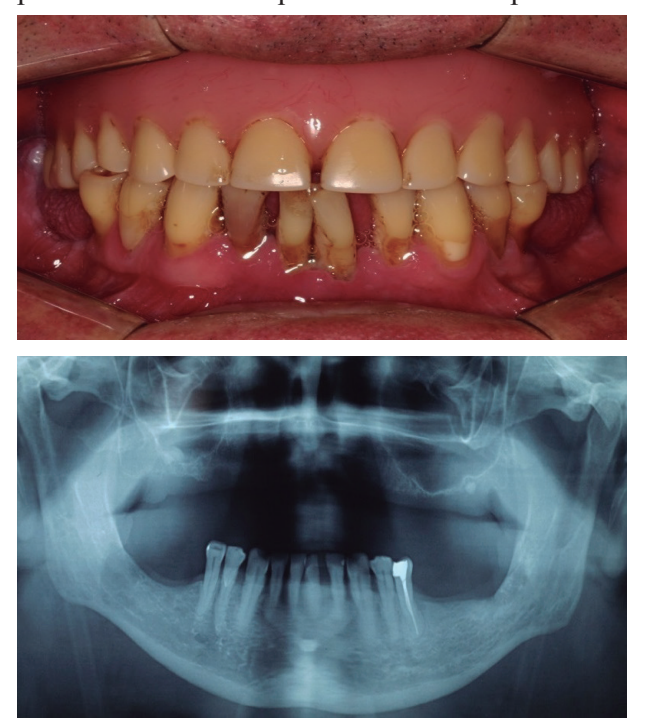

Fonte: Os autores.

Em consequência da necessidade de exodontias múltiplas em uma única sessão, foram necessários cuidados quanto ao aspecto psicológico do paciente por meio de diálogos, nos quais foram esclarecidas todas as dúvidas deste em relação à cirurgia e o pós-operatório, com o intuito de reduzir a ansiedade e estresse. Então, ele foi encaminhado para a residência de cirurgia buco-maxilo-facial para extrações dentárias e instalação dos cinco implantes. A radiografia panorâmica mostrou uma boa quantidade de osso mandibular anterior, portanto foi descartada a necessidade de enxerto ósseo (Figura 1).

Novas próteses totais, superior e inferior imediata foram confeccionadas utilizando dentes artificiais de resina acrílica Biolux ${ }^{\circledR}$ da cor 62 (Dental Vipi Ltda. Ind. Com. Imp. e Exp. de Produtos Odontológicos, Pirassununga-SP) com o projeto de extensão, intitulado "Reabilitação oral de pacientes com edentulismo total e parcial" da Universidade Estadual de Maringá. A confecção de novas próteses promove previsibilidade estética, guia para os implantes, além de fornecer um desenho mais harmônico destas que resulta em menos tensões geradas para a mandíbula, principalmente em relação ao protocolo de Bränemark ${ }^{13}$.
Figura 2: Cinco implantes foram instalados imediatamente após a extração dos dentes remanescentes
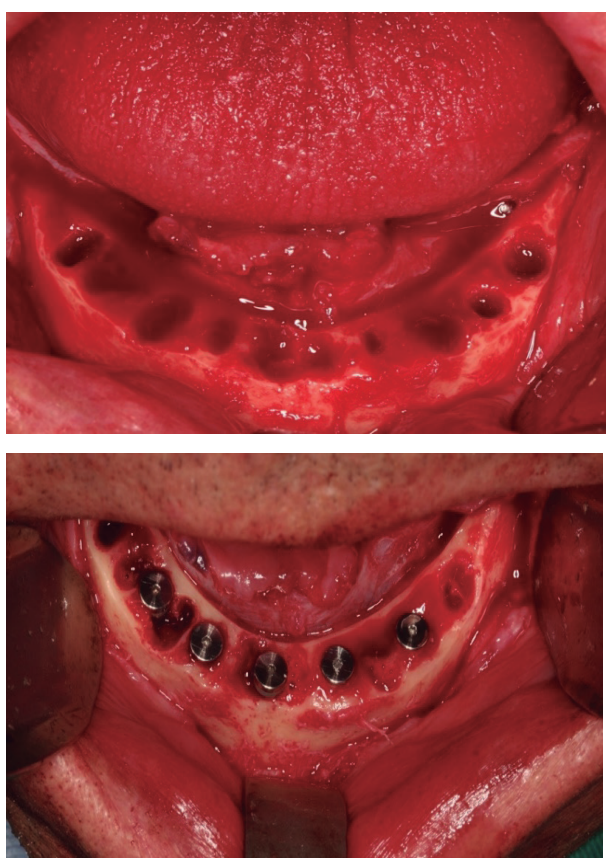

Fonte: Os autores.

Implantes da marca Neodent ${ }^{\circledR}$ (Neodent, Curitiba, Brasil) com hexágono externo que apresentavam plataforma 4.1 mm com $10 \mathrm{~mm}$ de comprimento cada (diâmetros maiores melhoram a distribuição de tensões $)^{14}$, foram eleitos para esse caso. Sabe-se que fumantes possuem níveis sistêmicos mais baixos de vitamina $C$, ainda que tenham um consumo similar a não fumantes ${ }^{15}$, e que a carência dessa vitamina afeta a produção de colágeno e dificulta a cicatrização. Como consequência desse fato, foi prescrita a suplementação com vitamina $\mathrm{C}$ um grama por 5 dias, antes da cirurgia e 5 dias após esta ${ }^{16}$.

Com esses cuidados prévios, a fase cirúrgia iniciou-se com anestesia infiltrativa com Mepivacaína 2\% associada à epinefrina 1:100.000, seguida da exodontia dos dentes inferiores, após a qual foi realizada incisão linear no rebordo e retalho total. Posteriormente, deu-se início às perfurações de acordo com recomendações da Neodent ${ }^{\circledR}$. O posicionamento dos implantes seguiu o guia cirúrgico previamente confeccionado e posição dos alvéolos. A instalação destes foram entre os forames mentonianos, de modo que os implantes mais distais ficassem a aproximadamente $4 \mathrm{~mm}$ do forame mentoniano, objetivando, assim, a preservação dos nervos alveolar inferior e mentoniano (Figura 2). As localizações dos implantes no arco influenciam na extensão posterior da barra metálica, pois em reabilitações totais da mandíbula, é recomendado que o cantilever deva ter no máximo 1,5 vezes o comprimento correspondente à distância entre os pontos mais posteriores dos implantes distais e o centro do implante localizado mais anteriormente no $\operatorname{arco}^{17}$. Adicionalmente a isso, os implantes foram posicionados da forma mais paralela 
possível entre si, a fim de facilitar a confecção da prótese e também fazendo com que cargas oclusais se direcionassem em seu longo eixo ${ }^{18}$. A quantidade, o tamanho e a posição destes definem também como as forças oclusais atuarão sobre estes e o osso ${ }^{14,19,20}$

Uma nova radiografia panorâmica mostrou a correta posição dos implantes na mandíbula. Nessa data aconteceu a substituição das tampas de proteção por cicatrizadores (Figura 3).

Figura 3: A radiografia panorâmica mostra o posicionamento dos implantes. Observação aos cicatrizadores instalados
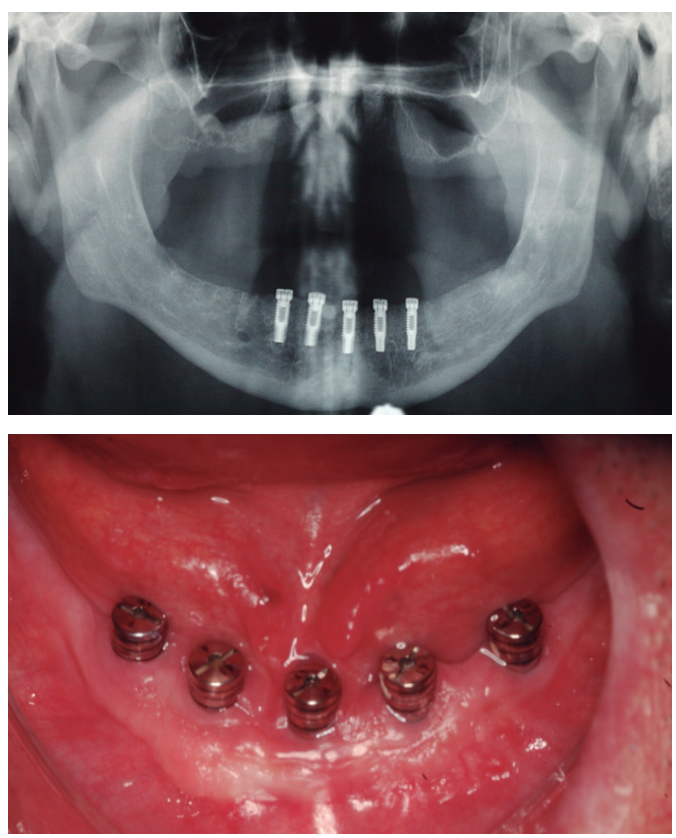

Fonte: Os autores.

O paciente permaneceu com a nova prótese total superior e a prótese inferior foi reembasada com material provisório Soft Confort $\AA$ (Dencril, Pirassununga, Brasil) até completar o período necessário para osseointegração satisfatória dos implantes (de 4 a 6 meses). Após seis meses, o paciente retornou, então foram iniciados os processos para se confeccionar a prótese inferior ${ }^{21}$. Este relatou dificuldade em usar a PT inferior reembasada, pois ela causava dor e incômodo.

Removendo os cicatrizadores, os parafusamentos dos cinco minipilares retos (Neodent, Curitiba, Brasil), com cinta de um milímetro cada (plataforma de 4.1), puderam ser feitos. Seguidamente uma radiografia periapical dos implantes e intermediários mostrou a adaptação correta destes, então foi aplicado um torque de $32 \mathrm{Ncm}$ com torquímetro em cada minipilar (Figura 4). A utilização de intermediários é de extrema relevância para uma melhor distribuição de tensões geradas durante a mastigação ${ }^{22}$, e o fato de os minipilares terem uma característica rotacional favorece o assentamento passivo de uma infraestutura metálica.
Figura 4: Cinco minipilares retos posicionados sobre as cabeças dos implantes
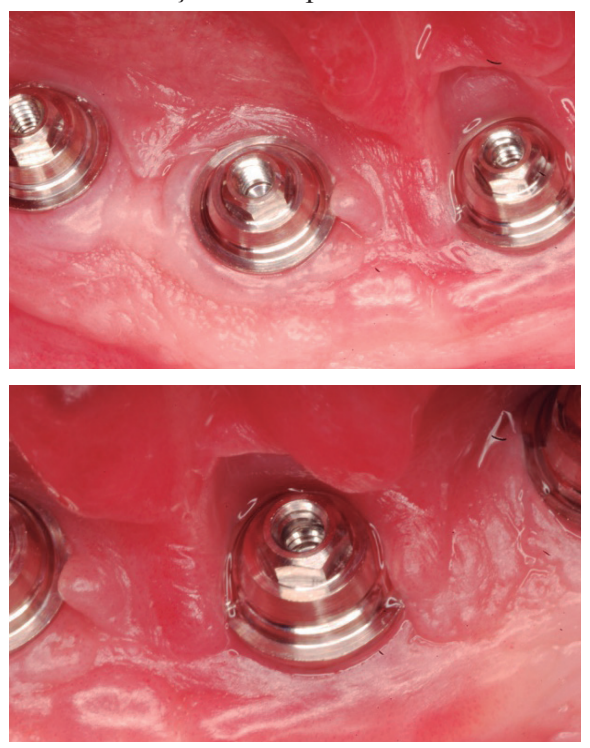

Fonte: Os autores.

Em seguida, cinco transferentes (Neodent, Curitiba, Brasil) de moldeira aberta foram posicionados e parafusados sobre os intermediários. Por meio da comprovação radiográfica e clínica da adaptação dos transferentes, estes foram unidos com fio dental e resina acrílica duralay (Reliance Dental Mfg, Worth, Ill., EUA) da cor vermelha. Concluída essa etapa, um período de 10 minutos foi aguardado até a completa polimerização da resina acrílica (Figura 5).

Figura 5: Vista dos passos para se conseguir a união com resina acrílica vermelha
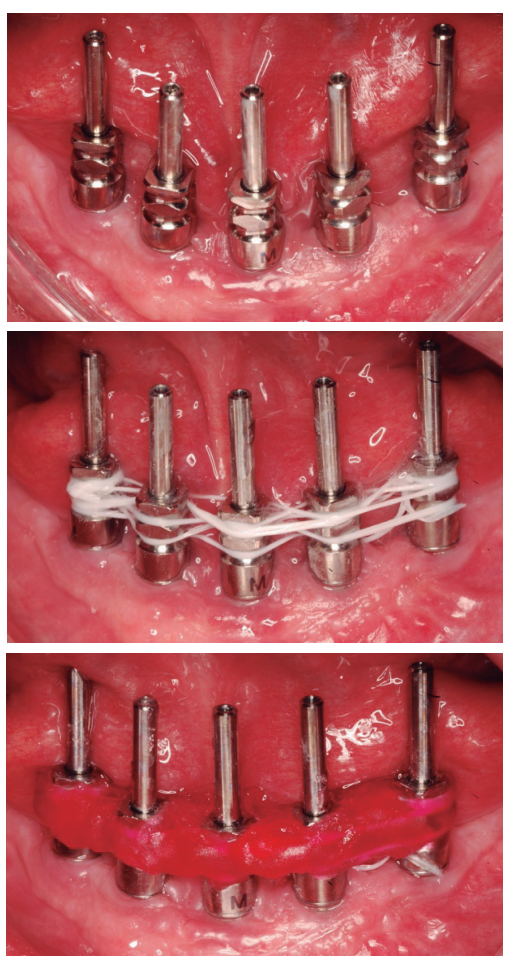

Fonte: Os autores. 
Com a silicona de adição leve e pesada Futura ${ }^{\circledR}(A D$, DFL, Brasil) iniciou-se a moldagem pela técnica da dupla mistura. Após 6 minutos, a moldeira foi removida da boca e os análogos foram posicionados no molde. Passado 2 horas (devido à liberação de hidrogênio) foi inserido à gengiva artificial Gingifast ${ }^{\circledR}$ (Zhermack, Itália), e o gesso especial Durone ${ }^{\circledR}$ tipo IV (Dentsply/Caulk,Milford, DE, USA) foi vazado obedecendo as recomendações do fabricante (Figura 6).

Figura 6: Molde obtido após soltar os parafusos e removê-lo da boca. Os análogos foram parafusados nos transferentes para receber o a gengiva artificial e o gesso especial Tipo IV
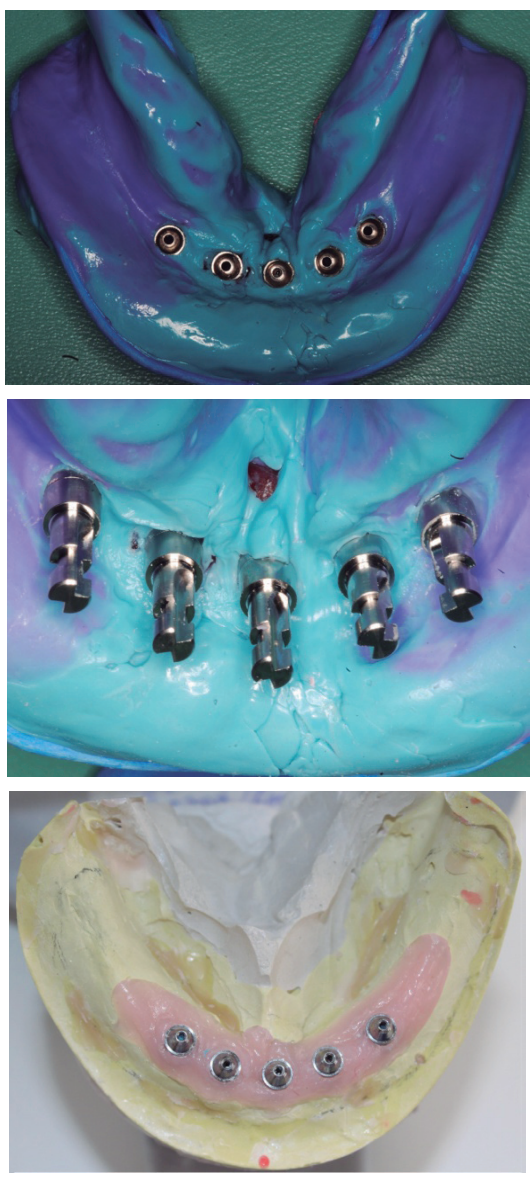

Fonte: Os autores.

Uma base de prova inferior com plano de orientação em cera foi confeccionada para se registrar a dimensão vertical de oclusão (DVO). Com o compasso de Willis foi estabelecida a DVO, que era igual à distância do canto do olho até a comissura labial (correspondente à dimensão vertical de repouso), menos três milímetros referentes ao espaço funcional livre. Essa DVO era semelhante à DVO que o paciente apresentava com suas próteses totais em oclusão.

O plano de cera inferior foi aquecido e introduzido novamente na cavidade bucal, assim a orientação de distância do compasso de Willis estabeleceria a DVO durante a oclusão. Com o registro em relação cêntrica concluído, uma moldagem da PT superior foi confeccionada em alginato (Cavex Color Change, Cavex Holland, Haarlem, Holanda) e o molde foi vazado com gesso pedra tipo III (Asfer®,Asfer Indústria Química, São Paulo). Depois da montagem dos modelos de gesso em articulador semiajustável (A7 FIX, Bioart, São Paulo, SP, Brasil), os dentes artificiais de resina acrílica Trilux ${ }^{\circledR}$ (Trilux EuroVIPI, VIPI, Pirassununga-SP, Brasil) inferiores puderam ser montados seguindo a escolha de cor pré-realizada com o paciente (Figura 7).

Figura 7: Imagem mostra o rolete de cera utilizado para o registro e o articulador semiajustável
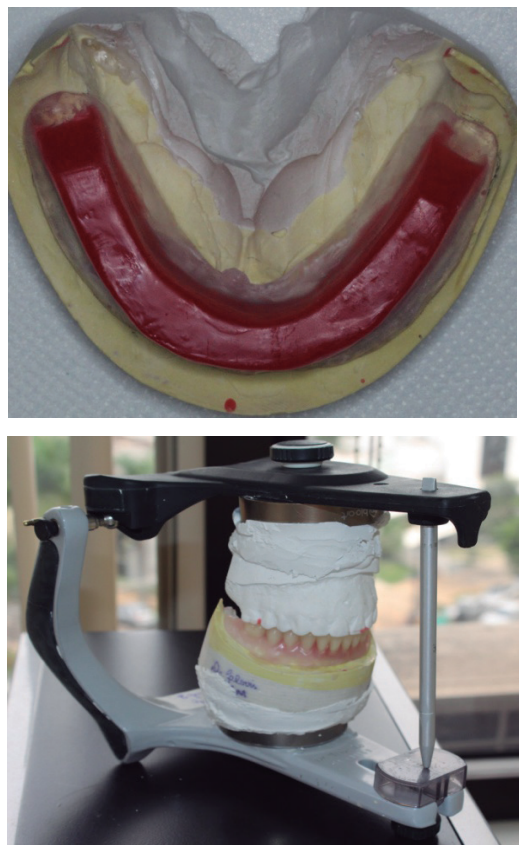

Fonte: Os autores.

Os dentes foram provados e ajustados na cavidade bucal, e nesse momento o acrílico da base de prova inferior foi colocado sobre os cicatrizadores dos minipilares, sem qualquer tipo de travamento aparafusado.

A montagem dos dentes da prótese inferior foi realizada até o primeiro molar seguindo o mesmo padrão de montagem da PT superior para se reduzir ao máximo a estrutura física do cantilever posterior e o excesso de forças sobre os implantes, principalmente os mais distais, que são vizinhos ao extremo livre ${ }^{13,22-24}$.

Após aprovação do paciente, a barra metálica de titânio foi confeccionada em CAD-CAM. Na figura 8, a extensão do cantilever foi de $18 \mathrm{~mm}$ aproximadamente de cada lado do arco, essa pouca extensão favorece a longevidade da saúde peri-implantar, a distribuição de cargas, além de evitar tensões exageradas nos implantes, nos componentes protéticos e na infraestrutura ${ }^{23-26}$. 
A prova da barra metálica foi passiva e a adaptação foi conferida por meio de radiografia periapical (Figura 8). A passividade é um fato relevante para o bom prognóstico da reabilitação em próteses sobre implantes, em que o excesso de forças e a má distribuição das tensões podem levar à falha dos implantes ${ }^{6}$.

Figura 8: Infraestrutura metálica assentada passivamente sobre os minipilares retos
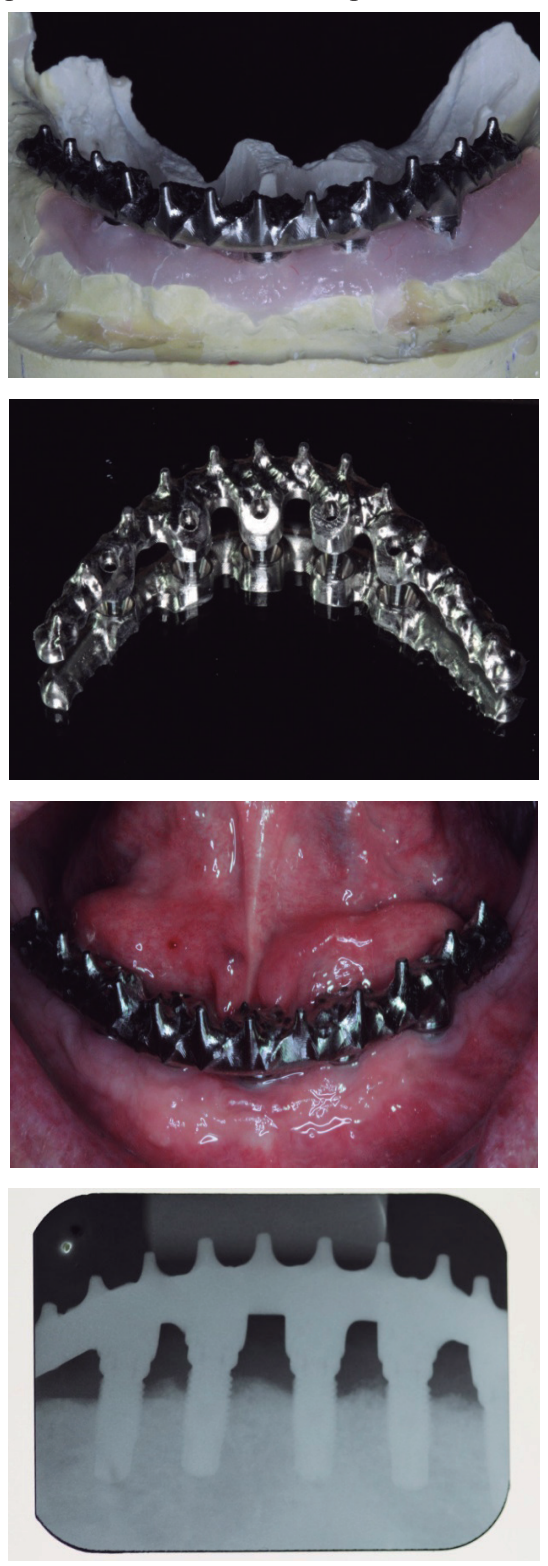

Fonte: Os autores.

Os dentes e a barra metálica foram unidos e ajustados na boca do paciente (Figura 9). Após verificação da oclusão, estética, cor da gengiva artificial (sistema Tomaz Gomes n ${ }^{\circ}$ : rosa normal) e adaptação, essa estrutura foi enviada para acrilização. Finalizada a acrilização, a prótese inferior foi instalada e os ajustes oclusais foram feitos apenas na prótese total superior (Figura 10).
Figura 9: Dentes artificiais e barra metálica foram unidos e ajustados na boca do paciente

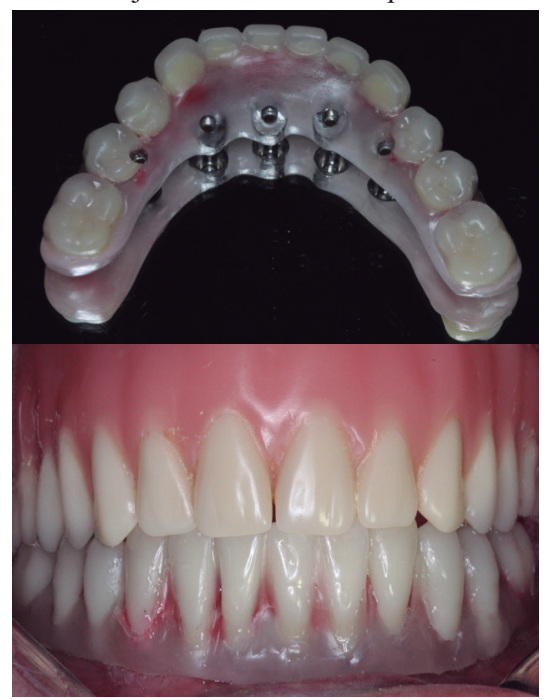

Depois da instalação da nova prótese sobre implantes, o paciente decidiu que gostaria de uma nova PT superior para melhorar ainda mais a estética de seu sorriso, bem como padronizar as tonalidades dos dentes e, para isso, a nova PT superior seguiu a mesma marca e matiz dos dentes inferiores (Trilux EuroVIPI, VIPI, Pirassununga-SP, Brasil).

Figura 10: Ajustes oclusais em oclusão, lateralidade e protrusão

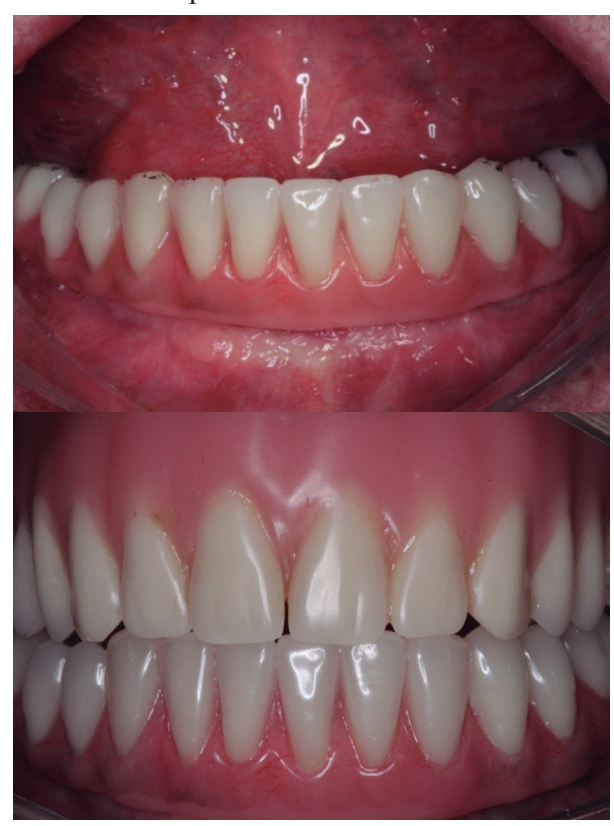

Fonte: Os autores.

Uma nova PT superior foi confeccionada pelo projeto de extensão "Reabilitação oral de pacientes com edentulismo total e parcial". O padrão de desoclusão pelo canino foi eleito ${ }^{27}$. $\mathrm{O}$ ajuste oclusal final foi feito até que 
cada dente tivesse pelo menos um contato simultâneo com seu antagonista. A posição do contato oclusal influencia diretamente no vetor da força, sendo assim, uma oclusão deficiente não proporciona um bom prognóstico.

Os parafusos protéticos receberam aproximadamente
$10 \mathrm{Ncm}$ de torque e foram vedados com fita veda rosca (politetrafluoretileno) e os orifícios com Bioplic ${ }^{\circledR}$ (Biodinâmica, Londrina, Brasil). Posteriormente o Bioplic foi substituído por resina composta micro-hibrida Z-250 (Z-250 XT -3M ESPE). A figura 11 mostra o antes e o depois do tratamento.

Figura 11: Condição clínica dos pacientes no início e após o término do tratamento
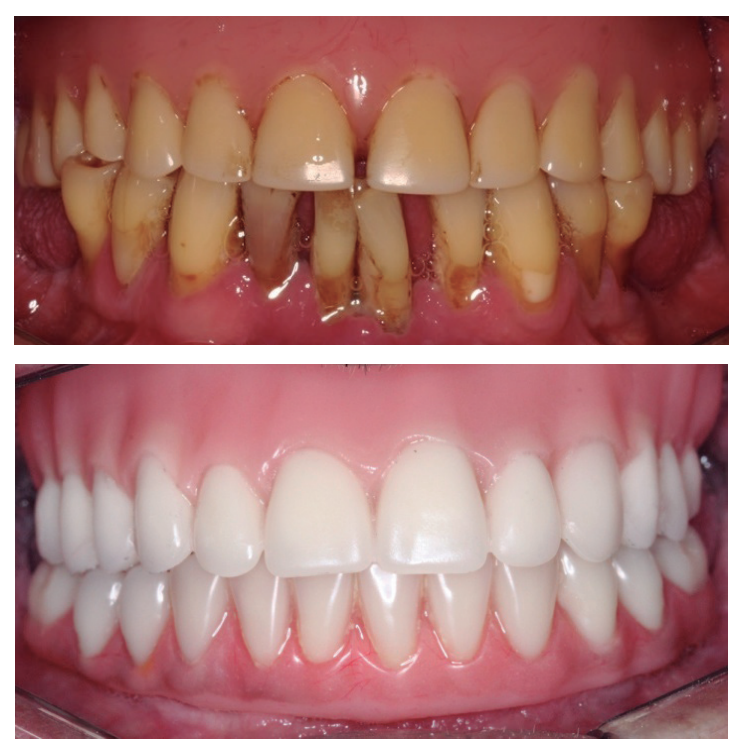

Fonte: Os autores.

Importante relatar que o paciente ao perceber uma mudança na estética inferior, se integrou mais ao tratamento, de modo a preferir o fechamento do diastema (Figura 12). Ressalta-se ainda que a nova PT superior obteve uma estética satisfatória, porque a PT superior anterior seguia bons parâmetros quanto à linha do sorriso, corredor bucal, curva de spee e tamanho dos dentes. Caso esses princípios não fossem respeitados, seria impossível confeccionar uma nova prótese com qualidade. Portanto, o cirurgião dentista deve saber julgar a qualidade do trabalho protético para evitar fracassos no tratamento reabilitador.

Figura 12: Condição estética apresentada durante e após o término do tratamento
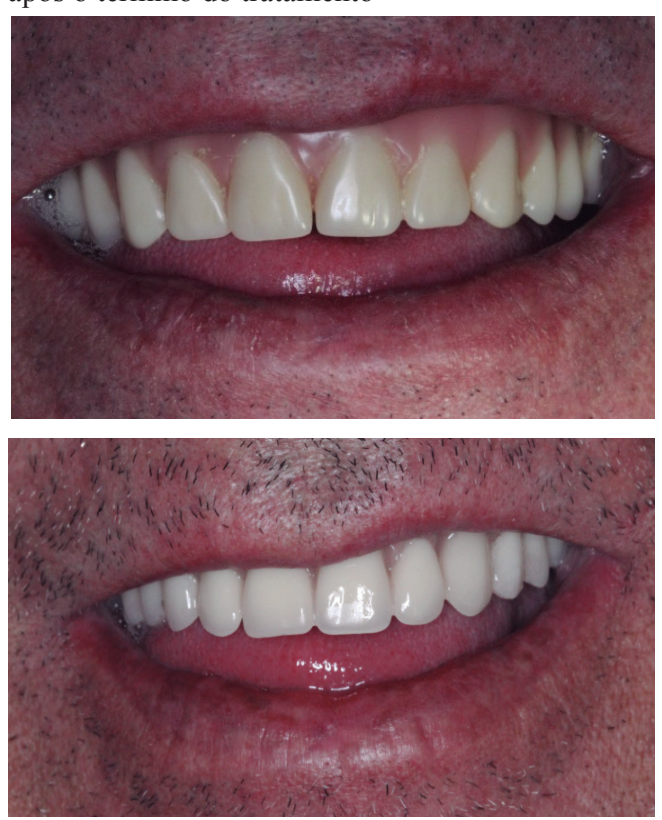

Fonte: Os autores. 
Foi reforçada a necessidade de cuidados bucais diários $^{28}$ com a prótese inferior e superior, a partir do uso de escovas dentais e interdentais, dieta, irrigador oral waterpik ${ }^{\circledR}$ e enxaguatórios bucais, além de retornos semestrais para acompanhamento. Essas medidas objetivam evitar o acúmulo de alimentos e placa bacteriana na região dos implantes e componentes protéticos, prevenindo, assim, o desenvolvimento de doenças peri-implantares. Em relação à higienização da prótese total superior, foi sugerida a escovação com sabão de coco para se evitar abrasão pelo creme dental e com isso aumentar a vida útil da prótese funcional e esteticamente. Posteriormente, o encaminhamento para um fonoaudiólogo foi efetivado para se obter uma adaptação da musculatura orofacial durante fala, mastigação e deglutição. Esse trabalho com outra área, além de ser de extrema importância no âmbito odontológico, ainda tem por objetivo a manutenção das condições emocionais e sociais para o indivíduo com novas próteses ${ }^{29}$. $\mathrm{O}$ fato da síndrome da combinação estar presente nesse caso enfatiza mais a necessidade da fonoaudiologia para que o paciente se acostume com a mastigação posterior e elimine esse hábito de concentrar esforços na região anterior do rebordo superior.

Apesar da prótese protocolo de resina ser uma alternativa para um tratamento de qualidade, ela tem um tempo de vida útil que varia conforme o tipo de alimentação, intensidade de mastigação e cuidados do paciente, pois os dentes em resina com o tempo vão sendo desgastados e consequentemente a dimensão vertical de oclusão reduzida. A partir disso, o paciente foi conscientizado durante todo o tratamento sobre essa questão de substituição de suas próteses com o tempo.

\section{Conclusão}

A condição socioeconômica é um fator que influencia no tipo do tratamento. Ao final da reabilitação, a satisfação do paciente se fez muito evidente com a melhora funcional e estética, assim este recuperou a autoestima e a vontade de cuidar melhor de sua saúde bucal. O planejamento com diferentes áreas da odontologia, associando a um projeto de extensão e a fonoaudiologia se mostrou de muita importância para esse caso.

\section{Referências}

1. Brasil. Ministério da Saúde. Secretaria de Atenção à Saúde. Secretaria de Vigilância em Saúde. SB Brasil 2010: Pesquisa Nacional de Saúde Bucal: resultados principais / Ministério da Saúde. Secretaria de Atenção à Saúde. Secretaria de Vigilância em Saúde. Brasília: MS; 2012.

2. Moy PK, Lundgren S, Holmes RE. Maxillary sinus augmentation: histomorphometric analysis of graft materials for maxillary sinus floor augmentation. J Oral Maxillo Fac Surg Philadelphia 1993;51(8):857-62.

3. Vaconcellos DK, Jóias RM, Volpato CAM, Blumer AB, Oliveira SHG. Disponibilidade óssea para instalação de implante na região edêntula de primeiro molar inferior. Odonto 2011;19(38):91-8.
4. Cardoso AC. O Passo a passo da prótese sobre implantes: da $2^{\mathrm{a}}$ etapa cirúrgica à reabilitação final. São Paulo: Santos; 2012.

5. Becker CM, Kaiser DA, Jones JD. Guidelines for splinting implants. J Prosthet Dent 2000;84(2):210-4.

6. Guichet DL, Caputo AA, Choi H, Sorensen JA. Passivity of fit marginal opening in screw - or cement - retained implants fixed partial denture designs. Int J Maxillo Fac Implants 2000;15(2):239-46.

7. Ng SKS, Chau AWL, Leung WK. The effect of properative information in relieving anxiety in oral surgery patients. Community Dent Oral Epidemiol 2004;32:227-35.

8. Renvert S, Polyzois IN. Clinical approaches to treat periimplant mucositis and peri-implantitis. Periodontology 2000;68:369-404.

9. Renvert S, Roos-Jansaker A-M, Claffey N. Non-surgical treatment of peri-implant mucositis and peri-implantitis: a literature review. J Clin Periodontol 2008;35(Suppl.8):305-15.

10. Toma S, Lasserre JF, Taïbe J, Brecx MC. Evaluation of an air-abrasive device with amino acid glycine-powder during surgical treatment of peri-implantitis. Quintessence Int 2004:45:209-19.

11. Portoriero R, Tonelli MP, Carnevale G, Mombelli A, Nyman SR, Lang NP. Experimentally induced peri-implant mucositis: a clinical study in humans. Clin Oral Implants Res 1994;5:254-9.

12. Heitz-Mayfield LJA. Peri-implant diseases: diagnosis and risk indicators. Clin Oral Implants Res 2008;35:214-22.

13. Rangert B, Jemt T, Jorneus L. Forces and moments on Branemark implants. Int $\mathrm{J}$ Oral Maxillofac Implants 1989;4(3):241-7.

14. Himmlová L, Dostálová T, Kácovský A, Konvicková S. Influence of implant length and diameter on stress distribution: a finite element analysis. J Prosthet Dent 2004;91:20-5.

15. Giraud DW, Martin HD, Driskell JA. Plasma and dietary vitamin $\mathrm{C}$ and $\mathrm{E}$ levels of tobacco chewers, smokers, and nonusers. J Am Diet Assoc 1995;95:798-800.

16. Tazima MFGS, Vicente YAMVA, Moriya T. Biologia da ferida e cicatrização. Medicina (Ribeirão Preto) 2008;41(3):259-64.

17. Wismeijer D, Buser D, Belser U. ITI Treatment Guide. Loading Protocols in Implant Dentistry. Edentulous Patients. Quintessence 2011.

18. Hsu YT, Fu JH, Al-Hezaimi K, Wang HL. Biomechanical implant treatment complications: a systematic review of clinical studies of implants with at least 1 year of functional loading. Int J Oral Maxillofac Implants 2012;27(4):894904.

19. .Lin CL, Wang JC, Kuo YC. Numerical simulation on the biomechanical interactions of tooth/implant-supported system under various occlusal forces with rigid/non-rigid connections. J Biomechanics 2006;3:453-63.

20. Misch CE. Contemporary implant dentistry. St Louis: MosbyYear Book; 1993.

21. Bränemark PI, Adell R, Breine J. Intraosseous anchorage of dental prostheses. Experimental studies. Scand. J Plast Reconstr.Surg Stockholm 1969;3(2):81-100.

22. Saeed R, Maryam K, Reza A, Mahdi K, Mohammad RM. Comparison of the effect of three abutment-implant connections on stress distribution at the internal surface of dental implants: a finite element analysis. J Dent Res Dent Clin Dent Prospects 2013;7(3):132-9. 
23. Greco GD, Greco ACDL, Greco IMGG, Seraidarian PI, Jansen WC. Cantilever in complete suported by implants. Revision of literature. Implant News 2008;5(6):627-32.

24. White SN, Dentsc MS, Caputo AA, Anderkvist T. Effect of cantilever length on stress transfer by implant-supported prostheses. J Prosthet Dent 1994;71(5):493-9.

25. Kökat AM, Cömert A, Tekdemir I, Akkocaoğlu M, Akça KI, Çehreli MC. Human ex vivo bone tissue strains around immediately-loaded implants supporting mandibular fixed prostheses. Implant Dent 2009;18(2):162-71.

26. Vasconcellos DK, Bottino MA, Nishioka RS, Luiz F, Valandro EMVC. The influence of different screw tightening forces on the vertical misfit of implant-supported frameworks. J Appl Oral Sci 2005;13(2).

27. Farias Neto A, Mestriner Junior W, AFP. Masticatory efficiency in denture wearers with bilateral balanced occlusion and canine guidance. Braz Dent J 2010;21(2).

28. Poluha RL, Melo Neto CLM, Sábio S. Prosthetic rehabilitation using association of total and implant-supported total denture (Brånemark protocol): case report. RSBO 2015;12(3):31622.

29. Cunha CC, Zuccolotto MCC, Bataglion C. Avaliação da eficiência mastigatória em paciente portador de prótese total. Rev APC 1999;53(3):214-7. 